\title{
Statement of Incoming Editor
}

I agreed to serve as editor of Animal Learning \& Behavior, not with the thought of creating a revolution, but with the hope of continuing the excellent traditions established by Abe Amsel, Mike D'Amato, Bob Bolles, Russ Church, Vin LoLordo, and Bob Rescorla. "If it ain't broke, don't fix it." Toward maintaining the high quality for which Animal Learning \& Behavior has become known, I have been fortunate enough to recruit Greg Fetterman, Marcia Spetch, and Bill Timberlake to serve as associate editors. Collectively, they will provide the editorial board with a breadth of expertise that no one person can possess.

My intention is to do, at most, a little fine tuning. Animal Learning \& Behavior will continue to publish primarily reports of programmatic empirical research concerning animal behavior that illuminate the nature of learning, memory, cognition, and motivation. Diverse theoretical orientations will continue to be welcome. But in addition to studies using traditional associative variables, I hope to see more work in an ethological vein (including behavior systems and evolutionary considerations) and papers that explore social stimuli.

Animal Learning \& Behavior will continue to periodically solicit review articles. However, unsolicited reviews, theoretical papers, and brief insightful commentaries will now also be considered for publication. Although multiple-experiment papers reporting programmatic research will continue to be the norm, well-controlled single-experiment studies that are sufficiently comprehensive to make a substantial contribution will also be considered. Moreover, I would like Animal Learning \& Behavior to publish more studies that examine animal learning and cognition in controlled naturalistic settings. And, although I anticipate that rats and pigeons will continue to serve as the subjects in most of the papers to be published, research done with other species, including humans, will be welcomed, provided that the manuscripts make firm contact with the existing animal learning and behavior literature.

In addition, I would like to see papers in Animal Learning \& Behavior that come from a greater variety of nations than is currently the case. One obstacle to this goal is that English is not the native language of many of the researchers in the field. Although all submissions should be written in as good English as possible, the staff of the Psychonomic Society Publications office can provide considerable assistance in correcting the grammar of accepted articles that originate in nonEnglish-speaking lands.

Finally, as a technical point, I am going to request that, whenever possible, manuscripts include some measure of variance for each reported measure of central tendency.

Through the support of the new set of associate and consulting editors and the continuing efforts of the many researchers who have in the past given generously of their time in writing thoughtful reviews, I anticipate that Animal Learning \& Behavior will continue to serve the field during the next 5 years as it has done so well in the past. 